\title{
Metabolite profiling and stable isotope tracing in sorted subpopulations of mammalian cells
}

\author{
Irena Roci ${ }^{1,2}$, Hector Gallart-Ayala ${ }^{3}$, Angelika Schmidt ${ }^{1,2}$, Jeramie Watrous ${ }^{4}$, Mohit Jain ${ }^{4}$, Craig E. \\ Wheelock ${ }^{3}$, Roland Nilsson ${ }^{1,2}$ \\ ${ }^{1}$ Karolinska Institutet, Department of Medicine, Unit of Computational Medicine \\ ${ }^{2}$ Karolinska Institutet, Center for Molecular Medicine \\ ${ }^{3}$ Karolinska Institutet, Department of Medical Biochemistry and Biophysics, Division of \\ Physiological Chemistry 2 \\ ${ }^{4}$ University of California San Diego, Department of Medicine
}

\begin{abstract}
Biological samples such as tissues, blood, or tumors are often complex and harbor heterogeneous populations of cells. Separating out specific cell types or subpopulations from such complex mixtures to study their metabolic phenotypes is challenging because experimental procedures for separation may disturb the metabolic state of cells. To address this issue, we developed a method for analysis of cell subpopulations using stable isotope tracing and fluorescence-activated cell sorting (FACS) followed by liquid chromatography - high resolution mass spectrometry (LC-HRMS). To ensure a faithful representation of cellular metabolism after cell sorting, we benchmarked sorted extraction against direct extraction. While peak areas differed markedly with lower signal for amino acids but higher signal for nucleotides, mass isotopomer distributions from sorted cells were generally in good agreement with those obtained from direct extractions, indicating that they reflect the true metabolic state of cells prior to sorting. In proof-of-principle studies, our method revealed metabolic phenotypes specific to $\mathrm{T}$ cell subtypes, and also metabolic features of cells in the committed phase of the cell division cycle. Our approach enables studies of a wide range of adherent and suspension cell subpopulations, which we anticipate will be of broad importance in cell biology and biomedicine.
\end{abstract}


a)
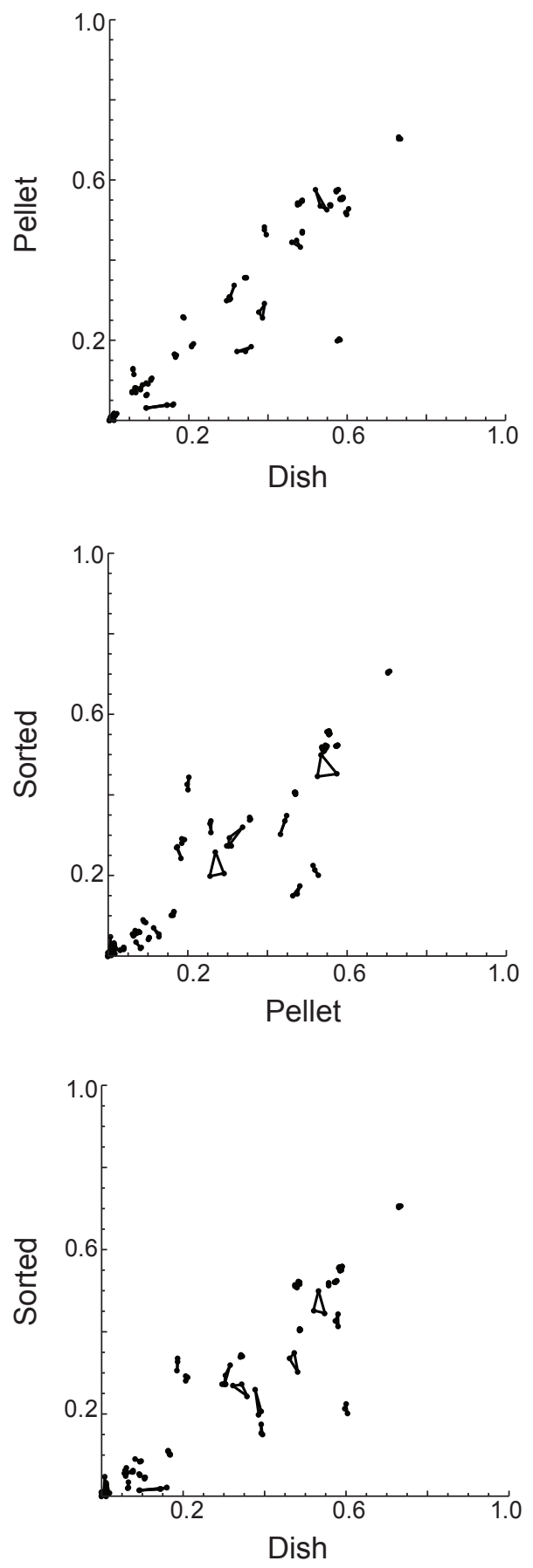

b)
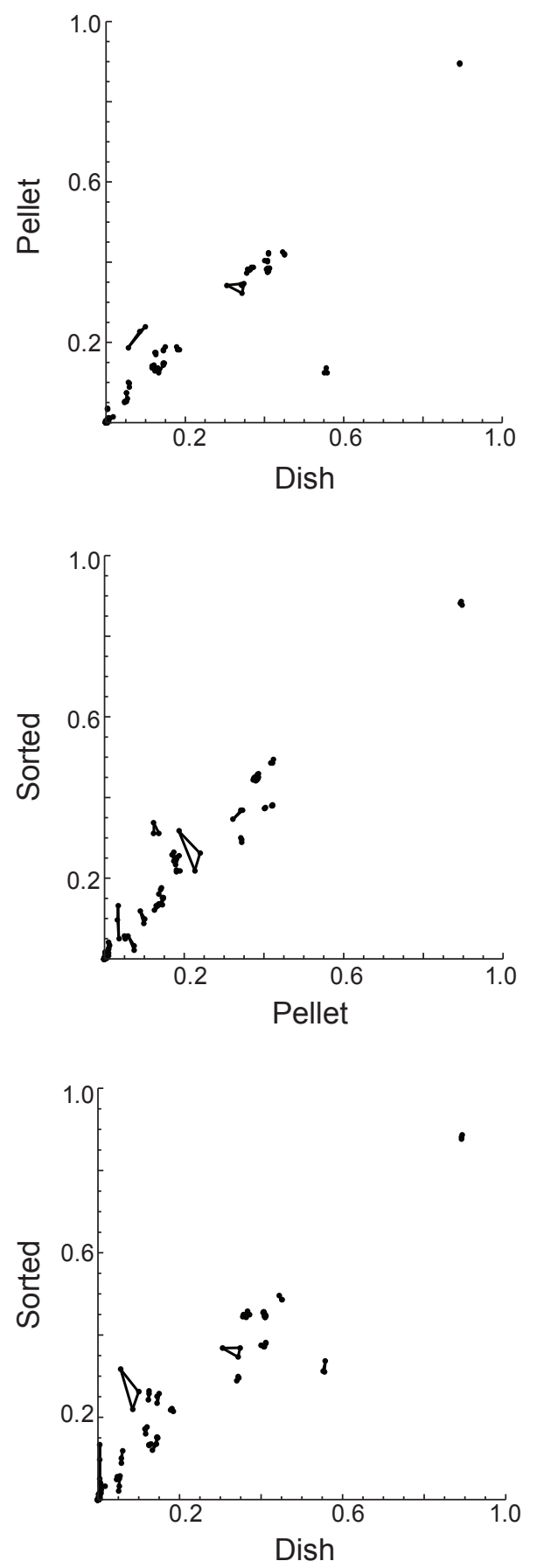

Figure S-1. Scatter plots of a $)^{13} \mathrm{C}$ and $\left.\mathrm{b}\right)^{15} \mathrm{~N}$ enrichment in dish, pellet and mock sorted samples. Each dot represents a replicate. Triplicates are joined with lines. 


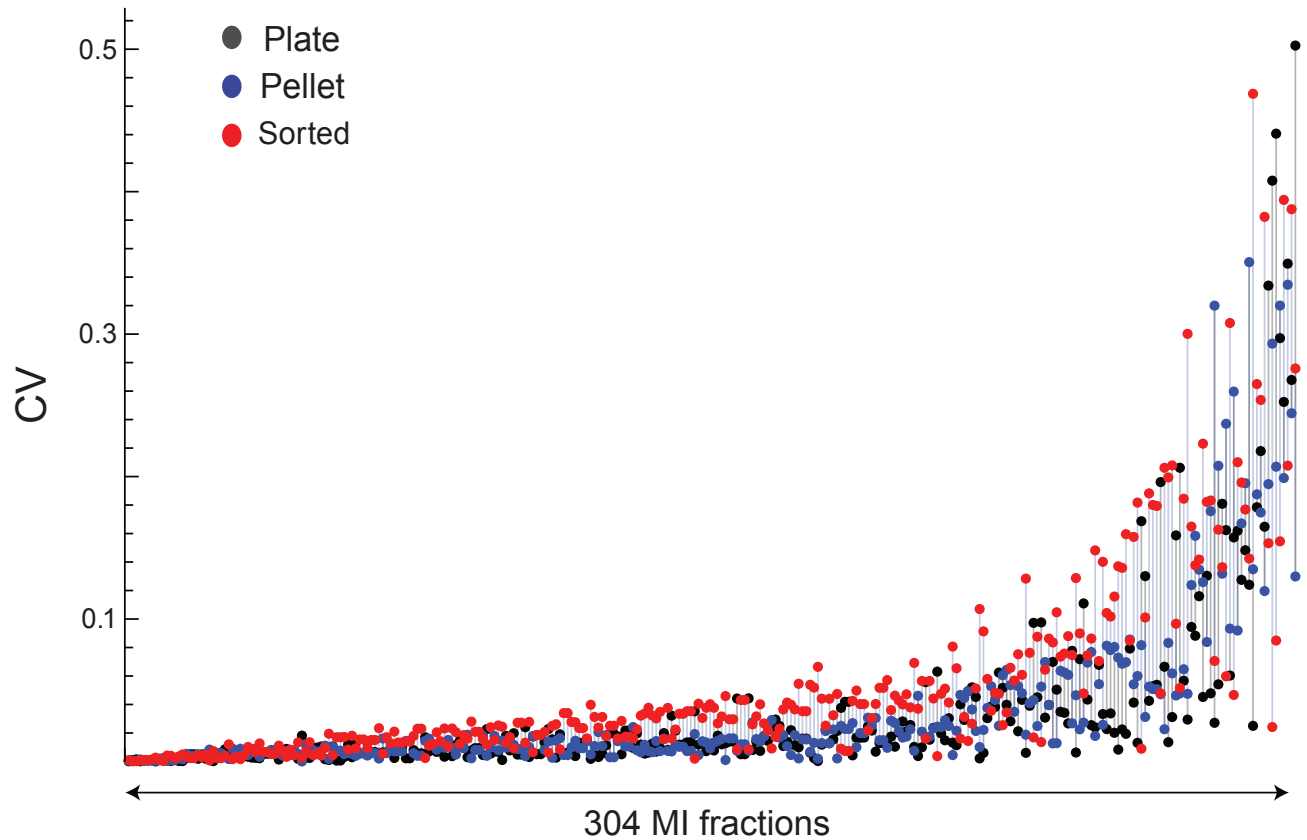

Figure S-2. CV of MI fractions with value lower than 0.01 . MI fractions of 61 metabolites which were below 0.01 were excluded from the calculation. 